\title{
A new small device made of glass for separating microplastics from marine and freshwater sediments
}

\author{
Ryota Nakajima ${ }^{\text {Corresp., } 1}{ }^{\text {, Masashi Tsuchiya }}{ }^{1}$, Dhugal J Lindsay ${ }^{1}$, Tomo Kitahashi ${ }^{1}$, Katsunori Fujikura ${ }^{1}$, Tomohiko \\ Fukushima ${ }^{1}$ \\ 1 Japan Agency for Marine-Earth Science and Technology, Yokosuka, Kanagawa, Japan \\ Corresponding Author: Ryota Nakajima \\ Email address: nakajimar@jamstec.go.jp
}

Separating microplastics from marine and freshwater sediments is challenging, but necessary to determine their distribution, mass and ecological impacts in benthic environments. Density separation is commonly used to extract microplastics from sediments by using heavy salt solutions, such as zinc chloride and sodium iodide. However, current devices/apparatus used for density separation, including glass beakers, funnels, upside-down funnel-shaped separators with a shut-off valve, etc., possess various shortcomings in terms of recovery rate, time consumption and/or usability. In evaluating existing microplastic extraction methods using density separation, we identified the need for a device that allows rapid, simple and efficient extraction of microplastics from a range of sediment types. We have developed a small glass separator, without a valve, taking a hint from an Utermöhl chamber. This new device is easy to clean and portable, yet enables rapid separation of microplastics from sediments. With this simple device, we recovered $94-98 \%$ of $<1,000 \mu \mathrm{m}$ microplastics (polyethylene, polypropylene, polyvinyl chloride, polyethylene terephthalate, and polystyrene). Overall, the device is efficient for various sizes, polymer types, and sediment types. Also, microplastics collected with this glassmade device remain chemically uncontaminated, and can therefore be used for further analysis of adsorbing contaminants and additives on/to microplastics. 


\title{
2 A new small device made of glass for separating
} 3 microplastics from marine and freshwater sediments

4

5

\author{
Ryota Nakajima ${ }^{1}$, Masashi Tsuchiya ${ }^{1}$, Dhugal J. Lindsay ${ }^{1}$, Tomo Kitahashi ${ }^{1}$, Katsunori \\ Fujikura $^{1}$, Tomohiko Fukushima ${ }^{1}$ \\ ${ }^{1}$ Japan Agency for Marine-Earth Science and Technology (JAMSTEC), Yokosuka, Kanagawa, \\ Japan
}

Corresponding Author:

Ryota Nakajima

2-15 Natsushima-cho, Yokosuka, Kanagawa 237-0061, Japan

Email address: nakajimar@jamstec.go.jp

\begin{abstract}
Separating microplastics from marine and freshwater sediments is challenging, but necessary to determine their distribution, mass and ecological impacts in benthic environments. Density separation is commonly used to extract microplastics from sediments by using heavy salt solutions, such as zinc chloride and sodium iodide. However, current devices/apparatus used for density separation, including glass beakers, funnels, upside-down funnel-shaped separators with a shut-off valve, etc., possess various shortcomings in terms of recovery rate, time consumption and/or usability. In evaluating existing microplastic extraction methods using density separation, we identified the need for a device that allows rapid, simple and efficient extraction of microplastics from a range of sediment types. We have developed a small glass separator, without a valve, taking a hint from an Utermöhl chamber. This new device is easy to clean and portable, yet enables rapid separation of microplastics from sediments. With this simple device, we recovered $94-98 \%$ of $<1,000 \mu \mathrm{m}$ microplastics (polyethylene, polypropylene, polyvinyl chloride, polyethylene terephthalate, and polystyrene). Overall, the device is efficient for various sizes, polymer types, and sediment types. Also, microplastics collected with this glass-made device remain chemically uncontaminated, and can therefore be used for further analysis of adsorbing contaminants and additives on/to microplastics.
\end{abstract}

\section{Introduction}

Microplastics, small pieces of plastic ranging from $5 \mathrm{~mm}$ in size down to microscopic, are ubiquitously distributed particulate contaminants that have to-date been detected in various 
40

41

42

43

44

45

46

47

48

49

50

51

52

53

54

55

56

57

58

59

60

61

62

63

64

65

66

67

68

69

70

71

72

73

74

75

76

77

78

79

environmental samples, including freshwater lakes and rivers (Eriksen et al., 2013; Free et al., 2014; Gasperi et al., 2014; Biginagwa et al., 2016), seas and oceans, including the deep-sea and the polar regions (Lusher et al., 2014; Shim \& Thomposon, 2015; Bergmann et al., 2017; Matsuguma et al., 2017; Wang et al., 2019). Once they end up in aquatic environments, microplastics eventually accumulate in/on the surface water, shores/beaches and in the sediments of benthic environments (Hidalgo-Ruz \& Thiel, 2013; Van Cauwenberghe et al., 2013; Law \& Thompson, 2014; Van Sebille et al., 2015). Marine and freshwater sediments are considered to possibly be the biggest sink of plastics in aquatic environments since significant numbers of microplastic fragments, films, spheres and fibers have been found in multiple sediment samples from various locations (Woodall et al., 2014; Bergmann et al., 2017; Matsuguma et al., 2017). However, we have not yet reached a consensus about the amounts of microplastics accumulating in oceanic and freshwater sediments, how these microplastics were transported into the sediments, and what their impact is on benthic animals (Farrell \& Nelson, 2013; Ugolini et al., 2013; Law \& Thompson, 2014; Green et al., 2016). Increased amounts of microplastics in/on the sediment enhance the risk of microplastic ingestion by benthic animals (Van Cauwenberghe et al., 2015a). Developing an efficient method for detecting microplastics from sediments is an important issue for further understanding the distribution, mass and ecological impacts of microplastics in both marine and freshwater environments.

One of the major concerns in microplastic studies of sediment samples is methodological - what is the best way to extract microplastics from a large amount of sediments? Several methods have been proposed to extract microplastics from sediment samples, including visual sorting, filtration, sieving, density separation, elutriation, flotation, chemical digestion, electrostatic behavior, and magnetic extraction (Hidalgo-Ruz et al., 2012; Anderson et al., 2015; Masura et al., 2015; Duis \& Coors, 2016; Felsing et al., 2018; Grbic et al., 2019). Density separation is a commonly used method to extract microplastics from sediments or sand using the principle of the difference in specific density of sediments (e.g. $\left.2.6 \mathrm{~g} \mathrm{~cm}^{-3}\right)$ and plastics $\left(0.1-1.7 \mathrm{~g} \mathrm{~cm}^{-3}\right)$ (Chubarenko et al., 2016). In density separation, dense solutions that have been proposed include sodium polytungstate (density: $\left.\sim 3.2 \mathrm{~g} \mathrm{~cm}^{-3}\right)$, zinc chloride $\left(\mathrm{ZnCl}_{2}\right.$, density: $\left.\sim 1.8 \mathrm{~g} \mathrm{~cm}^{-3}\right)$, zinc bromide ( $\mathrm{ZnBr}_{2}$, density: $1.7 \mathrm{~g} \mathrm{~cm}^{-3}$ ) and sodium iodide (NaI, density: $\sim 1.8 \mathrm{~g} \mathrm{~cm}^{-3}$ ) (Corcoran, Biesinger \& Grifi, 2009; Imhof et al., 2012; Liebezeit \& Dubaish, 2012; Dekiff et al., 2014; Van Cauwenberghe et al., 2015b; Quinn, Murphy \& Ewins, 2017; Prata et al., 2018). Due to the higher densities of these salt solutions compared to most microplastics $\left(0.9-1.5 \mathrm{~g} \mathrm{~cm}^{-3}\right)$, less dense microplastics float and separate out from the more dense, sinking, sediment materials (Quinn, Murphy \& Ewins, 2017).

During density separation a glass beaker is often used, but adhesion of microplastics onto the glass wall is a problem when pouring or sucking supernatant containing microplastics, thus requiring repeating the procedure. Avoiding resuspension of decanted sediments is also challenging when pouring supernatants (Masura et al., 2015). Use of a glass funnel is thus 
80

81

82

83

84

85

86

87

88

89

90

91

92

93

94

95

96

97

98

99

100

101

102

103

104

105

106

107

108

109

110

111

112

113

114

115

116

117

118

119

employed to avoid resuspension of sediments, but only a very small amount of sediment can be processed at any one time, requiring repeating the procedure (Masura et al., 2015). There is an upside-down funnel-shaped microplastic separator with a shut off valve (MPSS), but it is a large and heavy metal device and is thus not portable (Imhof et al., 2012). It also requires dismantling of the unit, including the shut off valve, for cleaning purposes between samples (Imhof et al., 2012). There is also a small and portable device based on the above large metal device (SMI), but it still requires cleaning of the shut off valve between samples (Coppock et al., 2017). Also, separators made of plastics (such as SMI) may not be appropriate for further analysis of adsorbing contaminants and additives on/to microplastics. In evaluating existing microplastic extraction devices using density separation, we identified the need for a device that allows rapid, simple and efficient extraction of microplastics from a range of sediment types. Here, we report a simple and small device made of glass, without a valve, for easier separation of microplastics from sediments.

\section{Materials \& Methods}

We developed a small separation unit made of glass without a valve, based loosely around a Utermöhl chamber (Utermöhl, 1931), that can isolate microplastics from sediments in a single step. The JAMSTEC microplastic-sediment separator (JAMSS) unit is constructed of two components - one upper and one lower modified glass plate (Fig. 1a). JAMSS can handle a variety of different sediment amounts by changing the volume of the tubes/containers attached to the lower and upper plates. Fig. $1 \mathrm{~b}$ shows small, middle and large models of JAMSS, consisting of a cylindrical container of $30 \mathrm{ml}, 60 \mathrm{ml}$, and $100 \mathrm{ml}$ volume, respectively. The upper plate incorporates an open glass tube, while the lower plate incorporates a cylindrical glass container (Fig. 1a). Each glass plate is frosted (ground) on the opposite side to that from which the respective tube or container is attached. The two plates are set against each other during use, initially so that the lower container and upper tube become a single, cylindrical container into which the sample can be poured (Fig. 1c). The plates can then be slid against each other to separate the lower and upper halves of the sample, respectively, by introducing a lid and bottom (the glass plate) to each half (Fig. 1d). The two plates stick together through the addition of a liquid to the frosted glass surface. Silicone grease can also be applied to the longitudinal edges of the glass plates to help them stick together. Metal clips can be attached to the plates for secure attachment when the plates are heavy/large (e.g. large plate in Fig. 1b).

\section{Microplastic separation from sediments}

With the JAMSS unit in its initial position, the internal walls of cylinders were thoroughly rinsed with distilled water prior to use. A sediment sample was placed in the lower cylindrical container using a spatula. Sodium iodide (NaI) solution was prepared by dissolving the salt in distilled water to achieve a final density of $1.6 \mathrm{~g} \mathrm{~cm}^{-3}$. We used $\mathrm{NaI}$ solution in this study because it is less environmentally dangerous compared to other commonly used salt solutions such as $\mathrm{ZnBr}_{2}$ 
120 (Quinn, Murphy \& Ewins, 2017). The solution was filtered through glass fiber filters (Whatman $121 \mathrm{GF} / \mathrm{F}$ ) prior to use in order to remove any contaminants. Then, the filtered NaI solution was 122 poured into the JAMSS unit so that the solution filled the cylinder to at least halfway up the 123 upper tube. A glass-covered magnetic stirring bar (VWR) was used to gently mix the sediment 124 for $20 \mathrm{~min}$ (Fig. 1e), and then the unit was left to settle until the supernatant was clear of

125

126

127

128

129

130

131

132

133

134

135

136

137

138

139

140

141

142

143

144

145

146

147

148

149

150

151

152

153

154

155

156

157

158

159

sediment. Next, the two plates were carefully slid so that the lower tubular container was completely sealed by the upper plate, ensuring there was no resuspension of sediments into the upper tube. The supernatant in the upper tube was then poured and filtered through a filter (such as a PTFE filter) by rinsing the internal walls of the tube with a squirt bottle filled with Milli-Q water (Millipore), in order to transfer all residual microplastics onto the filter (Fig. 1f). Filters were then transferred to a clean glass petri dish with a glass cover for further counting of microplastics. All the above procedures took place in a laminar flow hood, with the JAMSS unit being covered with aluminum foil when settling the sediment to minimize contamination.

\section{Validation of JAMSS}

To evaluate the extraction efficiencies of the JAMSS unit, first we tested the unit using finer sediments with known concentrations of microplastics of different polymer types and particle sizes. Secondly, we tested the unit using different sizes of sediment particles.

For the first experiment, we prepared five polymer types: polyethylene (PE, 0.92-0.97 $\mathrm{g} \mathrm{cm}^{-3}$ ), polypropylene (PP, 0.90-0.91 $\mathrm{g} \mathrm{cm}^{-3}$ ), polystyrene (PS, 0.04-1.09 $\mathrm{g} \mathrm{cm}^{-3}$ ), polyvinyl chloride (PVC, 1.35-1.45 $\mathrm{g} \mathrm{cm}^{-3}$ ) and polyethylene terephthalate (PET, 1.34-1.39 $\mathrm{g} \mathrm{cm}^{-3}$ ). These polymers were prepared in three size categories: $100-500 \mu \mathrm{m}, 500-1,000 \mu \mathrm{m}$ and 1,000-2,000 $\mu \mathrm{m}$ by milling plastic plates with a plastic-grinder (PLC-2M, Osaka Chemical) and then sizefractionated using stainless steel mesh screens of 100, 500, 1000 and $2000 \mu \mathrm{m}$ mesh size. We chose these three size categories as they are major size classes of microplastics found in sediments from various locations (Maes et al., 2017; Matsuguma et al., 2017; Peng et al., 2018; Mu et al., 2019; Zhang et al., 2019). We used fine-grained aquarium sand (ADA, Amazonica) as sediment, and this was ground beforehand using a mortar and pestle after adding Milli-Q water to moisten the sand to ease the grinding process. Prepared microplastics (40 particles per replicate) were mixed with $30 \mathrm{~g}$ sand (wet weight) in the unit and filtered $\mathrm{NaI}$ solution was then added. These added microplastics were distinguishable from the contaminants in the sand (mostly fibers), ensuring that only the added plastics were counted during the final enumeration. In total, five replicates of microplastic-sediment samples were examined for each plastic type and size. Microplastic-sediment samples were stirred in the JAMSS unit with a glass-covered magnetic stirrer as described above. Following filtering onto a membrane filter, the microplastics were counted under a stereomicroscope.

For the second experiment, we prepared reef carbonate sediments (Platinum reef sand, JUN company limited, Japan) in three size categories: fine $(31.9 \pm 93.8 \mu \mathrm{m})$, medium $(533.8 \pm 119.1$

Peer] reviewing PDF | (2019:06:38325:2:0:NEW 16 Sep 2019) 
160

161

162

163

164

165

166

167

168

169

170

171

172

173

174

175

176

177

178

179

180

181

182

183

184

185

186

187

188

189

190

191

192

193

194

195

196

197

198

199

$\mu \mathrm{m})$, and coarse $(1,165 \pm 212 \mu \mathrm{m})$. All the sediments were soaked in the NaI solution prior to use to remove possible contamination. PVC microplastics with a specific gravity of 1.35 (40 particles, size 500-1,000 $\mu \mathrm{m})$ were mixed with each sediment sample in the unit (10 g dry weight) and filtered $\mathrm{NaI}$ solution was then added. In total, five replicates of PVC microplasticsediment samples were examined for each sediment type, as described above.

The differences in the extraction efficiencies of microplastics between different polymer types or different particle sizes were determined using one-way ANOVA. The normality of the data and homogeneity of variance were examined and verified before ANOVA analysis using a chi-square test and a Bartlett test, respectively. When the one-way ANOVA was significant, differences among means were analyzed using Tukey-Kramer multiple comparison tests. A difference at $\mathrm{P}<0.05$ was considered significant.

\section{Results and Discussion}

Microplastics artificially incorporated into fine sand were extracted using the JAMSTEC microplastic-sedimentation separator (JAMSS) unit with $\mathrm{NaI}$ at a density of $1.6 \mathrm{~g} \mathrm{~cm}^{-3}$. No significant differences were found between the mean recovery rates of each different polymer type in any of the size categories $(\mathrm{P}=0.57$ for $100-500 \mu \mathrm{m}$ particles; $\mathrm{P}=0.25$ for $500-1000 \mu \mathrm{m}$ particles; $\mathrm{P}=0.06$ for $1,000-2,000 \mu \mathrm{m}$ particles, one-way ANOVA) (Fig. 2). When comparing the recovery rates of microplastics between the three size categories (i.e., 100-500 $\mu \mathrm{m}, 500-1000$ $\mu \mathrm{m}, 1,000-2,000 \mu \mathrm{m})$, overall mean recovery rates slightly decreased with decreasing size of the microplastic particles $(\mathrm{P}<0.0005$, one-way ANOVA) with significant differences between 100$500 \mu \mathrm{m}$ and 500-1,000 $\mu \mathrm{m}$ particles and 100-500 $\mu \mathrm{m}$ and $1,000-2,000 \mu \mathrm{m}$ particles $(\mathrm{P}<0.05$ Turkey-Kramer). These differences were probably because of the increased chance to lose smaller particles during processing of the samples (Nakajima et al., 2019). The recovery rates ranged from $92.5 \pm 5.6 \%$ to $96.0 \pm 4.2 \%$ for $100-500 \mu \mathrm{m}$ microplastics (overall mean $94.0 \pm$ $1.5 \%$ ), $96.5 \pm 2.9 \%$ to $100 \pm 0 \%$ for $500-1000 \mu \mathrm{m}$ microplastics (overall mean $97.8 \pm 1.3 \%$ ), and $97.8 \pm 1.6 \%$ to $100 \pm 0 \%$ for $1000-2000 \mu \mathrm{m}$ microplastics (overall mean $99.1 \pm 1.0 \%$ ). The recovery rates for $<1,000 \mu \mathrm{m}$ microplastics (94.0-97.8\%) were comparable with those of previously developed separators, $95.5 \%$ for MPSS (Imhof et al., 2012) and 95.8\% for SMI (Coppock et al., 2017).

Microplastics incorporated into different granularities of reef sediment (fine, $32 \mu \mathrm{m}$; medium, $534 \mu \mathrm{m}$; coarse, $1,165 \mu \mathrm{m})$ revealed the JAMSS unit extracted microplastics efficiently $(96.0 \pm$ $3.4 \%$ for fine sand, $98.5 \pm 1.4 \%$ for medium sand, $97.0 \pm 1.1 \%$ for coarse sand) (Fig. 3). No significant differences ( $\mathrm{p}=0.23$, one-way ANOVA) were found between the three grain sizes, showing efficient microplastic separation out from sediments using heavy salt solution (NaI), regardless of grain size. 
200

201

202

203

204

205

206

207

208

209

210

211

212

213

214

215

216

217

218

219

220

221

222

223

224

225

226

227

228

229

230

231

232

233

234

235

236

237

238

239

Among the previous methods for density separation of microplastics, the classical decanting method, for example the use of a beaker, is simple in design but adhesion of microplastics to the inside of the container is a problem when the media is transferred, thus resulting in a relatively low recovery rate $(40 \%)$ (Imhof et al., 2012). The technique is often repeated 3-5 times to raise the extraction efficiency, but this extends the processing time for each sample and increases the chance to lose microplastic samples (Nakajima et al., 2019). Avoiding resuspension of decanted sediments is also challenging when pouring supernatant (Masura et al., 2015). Conversely, JAMSS completely seals the sediment in a closed space (i.e. the lower container) therefore stopping the introduction of resuspended sediments into the supernatant. The microplasticcontaining supernatant can then be easily transferred by rinsing the inside walls of the unit, allowing high extraction efficiency of microplastics in a single step.

To combat the weak points of the classical decanting method, use of a glass funnel can be employed to avoid resuspension of sediments, but only a very small amount of sediment can be processed at any one time, requiring repeating the procedure (Masura et al., 2015). The device is also easily clogged irrevocably when processing relatively large, coarse sediments. In contrast, JAMSS can handle a variety of different sediment amounts by changing the volume of the tubes/containers attached to the lower and upper plates (Fig. 1b). Currently the large model of JAMSS consists of a cylindrical container of $100 \mathrm{ml}$ volume, allowing direct placement of the entire sediment content of a core sample slice (e.g. $10 \mathrm{~mm}$ thickness, $80 \mathrm{~mm}$ diameter).

Previously, an upside-down funnel-shaped microplastic separator with a shut off valve (MPSS) had been proposed as the best method for separating microplastics from sediment samples, but this is a large and heavy metal device and is thus not portable (Imhof et al., 2012). It also requires dismantling of the unit, including the shut off valve, for cleaning purposes between samples (Imhof et al., 2012). There is also a small, portable device based on the above large metal device (SMI), but it still requires cleaning of the shut off valve between processing samples (Coppock et al., 2017). From this point of view, JAMSS has no valve and has a simple structure, allowing rapid cleaning and reductions in sample processing times.

Though SMI is a portable unit (Coppock et al., 2017), it is made of plastic (PVC), thus it may not be appropriate for further analysis of adsorbing persistent organic pollutants (POPs) and other chemicals such as additives on/to microplastics. One of the advantages of the JAMSS is that it is made of glass, so microplastics remain uncontaminated for use in further chemical analyses.

Overall, the JAMSS unit is a small, portable device that enables the extraction of microplastics from sediments in a single step. It is simple, without a shut-off valve, and is thus easy to use. The JAMSS has also proven compatible with both finer sediments and coarse sand. Because of the size of this small device, all the above procedures can be done in a laminar flow hood. It should be noted, however, that when the sediment is clay-like or otherwise interferes with the rotation of 
240 the magnetic stirrer bar, dilution with water may be necessary. Microplastics can also get caught

241 in the silicone grease used for lubrication between the two plates, so the grease should only be

242 applied to the longitudinal edges of the plates, which do not come into contact with the sample.

243

244

245

246

247

We have developed a small, glass, microplastic-sedimentation separator (JAMSS), without a shut

248 off valve, taking a hint from an Utermöhl chamber. This new device is easy to clean and portable, yet enables rapid separation of microplastics from sediments in a single step. In using

250

251

252

253

254

255

256

257

258

259

260

261

262

263

264

265

266

267

268

269

270

271

272

273

274

275

276

277

278

279

280

281

282 the JAMSS unit, the user has the advantage of being able to rinse the entire headspace multiple times without resuspending the settled sediment, therefore reducing the need for repetitive processing and limiting the possibilities of external contamination and loss of microplastics. The lack of a shut off valve means there is no chance of it becoming clogged. Unlike devices made of plastic, microplastics separated from sediments with this glass-made JAMSS unit remain chemically uncontaminated so they can be used for further analysis of adsorbing contaminants and additives on/to microplastics.

\section{Acknowledgements}

We thank Rie Matsui for her help in validating the unit.

\section{References}

Anderson A, Andrady A, Arthur C, Baker J, Bouwman H, Gall S, Hildalgo-Ruz V, Köhler A, Lavender Law K, Leslie HA. 2015. Sources, fate and effects of microplastics in the environment: a global assessment. GESAMP Reports \& Studies Series.

Bergmann M, Wirzberger V, Krumpen T, Lorenz C, Primpke S, Tekman MB, Gerdts G. 2017. High quantities of microplastic in Arctic deep-sea sediments from the HAUSGARTEN observatory. Environmental science \& technology 51:11000-11010.

Biginagwa FJ, Mayoma BS, Shashoua Y, Syberg K, Khan FR. 2016. First evidence of microplastics in the African Great Lakes: recovery from Lake Victoria Nile perch and Nile tilapia. Journal of Great Lakes Research 42:146-149.

Chubarenko I, Bagaev A, Zobkov M, Esiukova E. 2016. On some physical and dynamical properties of microplastic particles in marine environment. Marine Pollution Bulletin 108:105-112.

Coppock RL, Cole M, Lindeque PK, Queirós AM, Galloway TS. 2017. A small-scale, portable method for extracting microplastics from marine sediments. Environmental Pollution 230:829-837.

Corcoran PL, Biesinger MC, Grifi M. 2009. Plastics and beaches: a degrading relationship. Marine Pollution Bulletin 58:80-84.

Dekiff JH, Remy D, Klasmeier J, Fries E. 2014. Occurrence and spatial distribution of microplastics in sediments from Norderney. Environmental Pollution 186:248-256.

Duis K, Coors A. 2016. Microplastics in the aquatic and terrestrial environment: sources (with a specific focus on personal care products), fate and effects. Environmental Sciences Europe 28:2. 
283

284

285

286

287

288

289

290

291

292

293

294

295

296

297

298

299

300

301

302

303

304

305

306

307

308

309

310

311

312

313

314

315

316

317

318

319

320

321

322

323

324

325

326

Eriksen M, Mason S, Wilson S, Box C, Zellers A, Edwards W, Farley H, Amato S. 2013. Microplastic pollution in the surface waters of the Laurentian Great Lakes. Marine pollution bulletin 77:177-182.

Farrell P, Nelson K. 2013. Trophic level transfer of microplastic: Mytilus edulis (L.) to Carcinus maenas (L.). Environmental pollution 177:1-3.

Felsing S, Kochleus C, Buchinger S, Brennholt N, Stock F, Reifferscheid G. 2018. A new approach in separating microplastics from environmental samples based on their electrostatic behavior. Environmental pollution 234:20-28.

Free CM, Jensen OP, Mason SA, Eriksen M, Williamson NJ, Boldgiv B. 2014. High-levels of microplastic pollution in a large, remote, mountain lake. Marine pollution bulletin $85: 156-$ 163.

Gasperi J, Dris R, Bonin T, Rocher V, Tassin B. 2014. Assessment of floating plastic debris in surface water along the Seine River. Environmental pollution 195:163-166.

Grbic J, Nguyen B, Guo E, You JB, Sinton D, Rochman CM. 2019. Magnetic Extraction of Microplastics from Environmental Samples. Environmental Science \& Technology Letters 6:68-72.

Green DS, Boots B, Sigwart J, Jiang S, Rocha C. 2016. Effects of conventional and biodegradable microplastics on a marine ecosystem engineer (Arenicola marina) and sediment nutrient cycling. Environmental Pollution 208:426-434.

Hidalgo-Ruz V, Gutow L, Thompson RC, Thiel M. 2012. Microplastics in the marine environment: a review of the methods used for identification and quantification. Environmental science \& technology 46:3060-3075.

Hidalgo-Ruz V, Thiel M. 2013. Distribution and abundance of small plastic debris on beaches in the SE Pacific (Chile): a study supported by a citizen science project. Marine environmental research 87:12-18.

Imhof HK, Schmid J, Niessner R, Ivleva NP, Laforsch C. 2012. A novel, highly efficient method for the separation and quantification of plastic particles in sediments of aquatic environments. Limnology and oceanography: methods 10:524-537.

Law KL, Thompson RC. 2014. Microplastics in the seas. Science 345:144-145.

Liebezeit G, Dubaish F. 2012. Microplastics in beaches of the East Frisian islands Spiekeroog and Kachelotplate. Bulletin of Environmental Contamination and Toxicology 89:213-217.

Lusher AL, Burke A, O’Connor I, Officer R. 2014. Microplastic pollution in the Northeast Atlantic Ocean: validated and opportunistic sampling. Marine pollution bulletin 88:325333.

Maes T, Van der Meulen MD, Devriese LI, Leslie HA, Huvet A, Frère L, Robbens J, Vethaak AD. 2017. Microplastics baseline surveys at the water surface and in sediments of the North-East Atlantic. Frontiers in Marine Science 4:135.

Masura J, Baker JE, Foster GD, Arthur C, Herring C. 2015. Laboratory methods for the analysis of microplastics in the marine environment: recommendations for quantifying synthetic particles in waters and sediments. NOAA Tech. Memo. NOS-OR\&R-48.

Matsuguma Y, Takada H, Kumata H, Kanke H, Sakurai S, Suzuki T, Itoh M, Okazaki Y, Boonyatumanond R, Zakaria MP. 2017. Microplastics in sediment cores from Asia and Africa as indicators of temporal trends in plastic pollution. Archives of environmental contamination and toxicology 73:230-239.

Peer) reviewing PDF | (2019:06:38325:2:0:NEW 16 Sep 2019) 
327

328

329

330

331

332

333

334

335

336

337

338

339

340

341

342

343

344

345

346

347

348

349

350

351

352

353

354

355

356

357

358

359

360

361

362

363

364

365

366

367

Mu J, Qu L, Jin F, Zhang S, Fang C, Ma X, Zhang W, Huo C, Cong Y, Wang J. 2019. Abundance and distribution of microplastics in the surface sediments from the northern Bering and Chukchi Seas. Environmental pollution 245:122-130.

Nakajima R, Lindsay DJ, Tsuchiya M, Matsui R, Kitahashi T, Fujikura K, Fukushima T. 2019. A small, stainless-steel sieve optimized for laboratory beaker-based extraction of microplastics from environmental samples. Methods $X$.

Peng X, Chen M, Chen S, Dasgupta S, Xu H, Ta K, Du M, Li J, Guo Z, Bai S. 2018. Microplastics contaminate the deepest part of the world's ocean. Geochemical Perspectives Letters 9:1-5.

Prata JC, da Costa JP, Duarte AC, Rocha-Santos T. 2018. Methods for sampling and detection of microplastics in water and sediment: a critical review. TrAC Trends in Analytical Chemistry.

Quinn B, Murphy F, Ewins C. 2017. Validation of density separation for the rapid recovery of microplastics from sediment. Analytical Methods 9:1491-1498.

Van Sebille E, Wilcox C, Lebreton L, Maximenko N, Hardesty BD, Van Franeker JA, Eriksen M, Siegel D, Galgani F, Law KL. 2015. A global inventory of small floating plastic debris. Environmental Research Letters 10:124006.

Shim WJ, Thomposon RC. 2015. Microplastics in the ocean. Archives of environmental contamination and toxicology 69:265-268.

Ugolini A, Ungherese G, Ciofini M, Lapucci A, Camaiti M. 2013. Microplastic debris in sandhoppers. Estuarine, Coastal and Shelf Science 129:19-22.

Utermöhl H. 1931. Neue wege in der quantitativen Erfassung des planktons. Verh. Int. Ver. Limnol. 5:567-596.

Van Cauwenberghe L, Claessens M, Vandegehuchte MB, Janssen CR. 2015a. Microplastics are taken up by mussels (Mytilus edulis) and lugworms (Arenicola marina) living in natural habitats. Environmental Pollution 199:10-17.

Van Cauwenberghe L, Devriese L, Galgani F, Robbens J, Janssen CR. 2015b. Microplastics in sediments: a review of techniques, occurrence and effects. Marine environmental research 111:5-17.

Van Cauwenberghe L, Vanreusel A, Mees J, Janssen CR. 2013. Microplastic pollution in deepsea sediments. Environmental pollution 182:495-499.

Wang J, Wang M, Ru S, Liu X. 2019. High levels of microplastic pollution in the sediments and benthic organisms of the South Yellow Sea, China. Science of The Total Environment 651:1661-1669.

Woodall LC, Sanchez-Vidal A, Canals M, Paterson GLJ, Coppock R, Sleight V, Calafat A, Rogers AD, Narayanaswamy BE, Thompson RC. 2014. The deep sea is a major sink for microplastic debris. Royal Society open science 1:140317.

Zhang C, Zhou H, Cui Y, Wang C, Li Y, Zhang D. 2019. Microplastics in offshore sediment in the Yellow Sea and East China Sea, China. Environmental pollution 244:827-833. 


\section{Figure 1}

JAMSTEC microplastic-sedimentation separator (JAMSS) unit

(a) the upper plate (left) incorporates an open glass tube, while the lower plate (right) incorporates a cylindrical glass container. (b) small, middle and large models of assembled JAMSS, consisting of a cylindrical container of $30 \mathrm{ml}, 60 \mathrm{ml}$, and $100 \mathrm{ml}$ volume, respectively. (c) JAMSS during density floatation with sediment in the lower container. (d) separation of sediment and supernatant by sliding the two plates against each other. (e) JAMSS can be placed on a magnetic stirrer to ensure the sediments are well mixed during microplastic floatation. (f) microplastics in the supernatant in the upper tube are poured by rinsing the internal walls of the tube. 

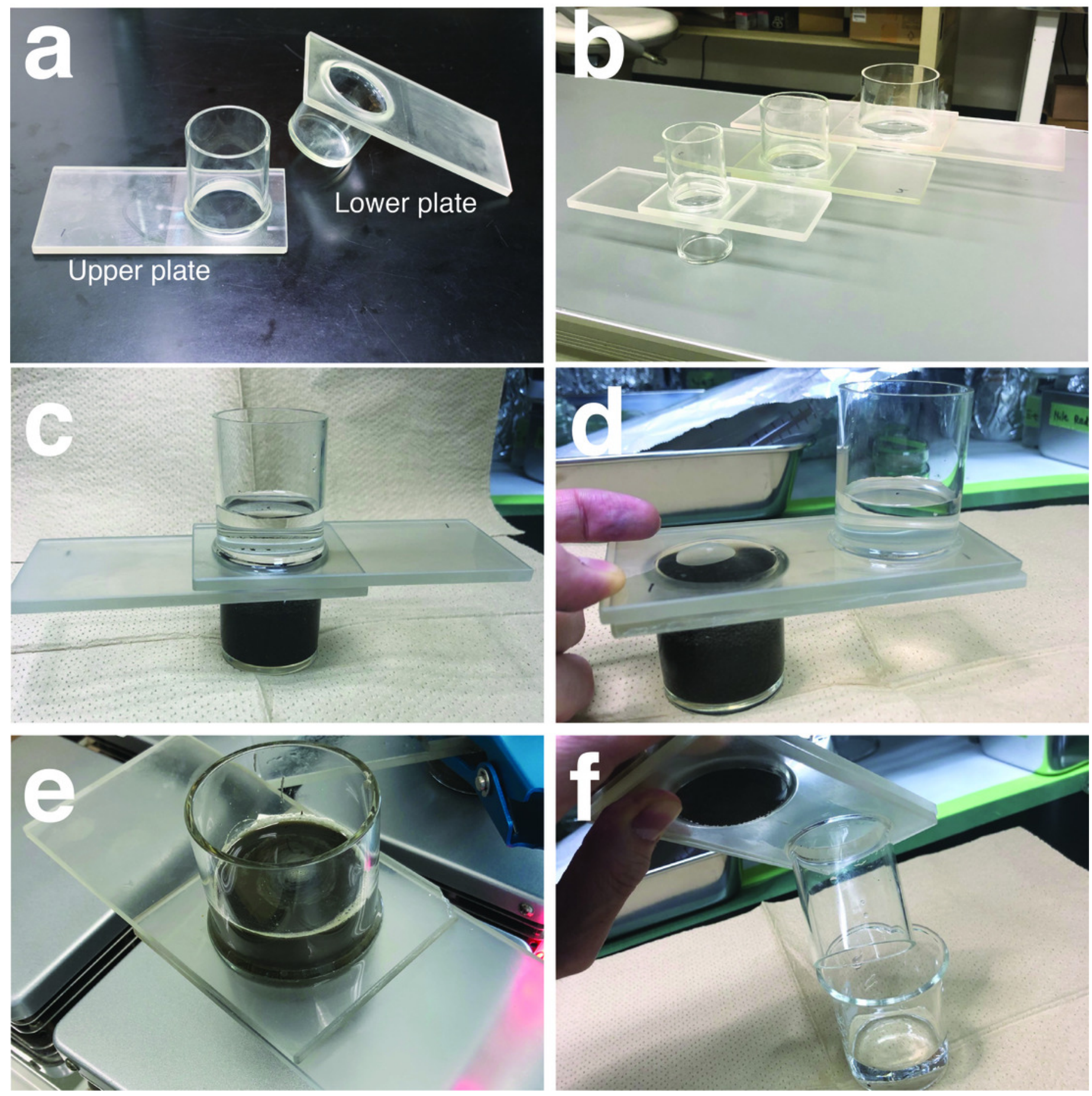
Figure 2

Recovery rate (\%) of microplastics of different polymer types and particle sizes by JAMSS unit

Each bar indicates the average recovery rates of microplastics of different size particles: (a)

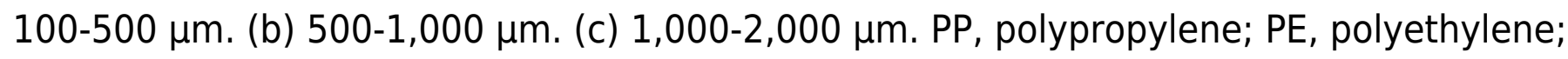

PET, polyethylene terephthalate; PS, polystyrene; PVC, polyvinyl chloride. Error bars indicate standard deviation. 


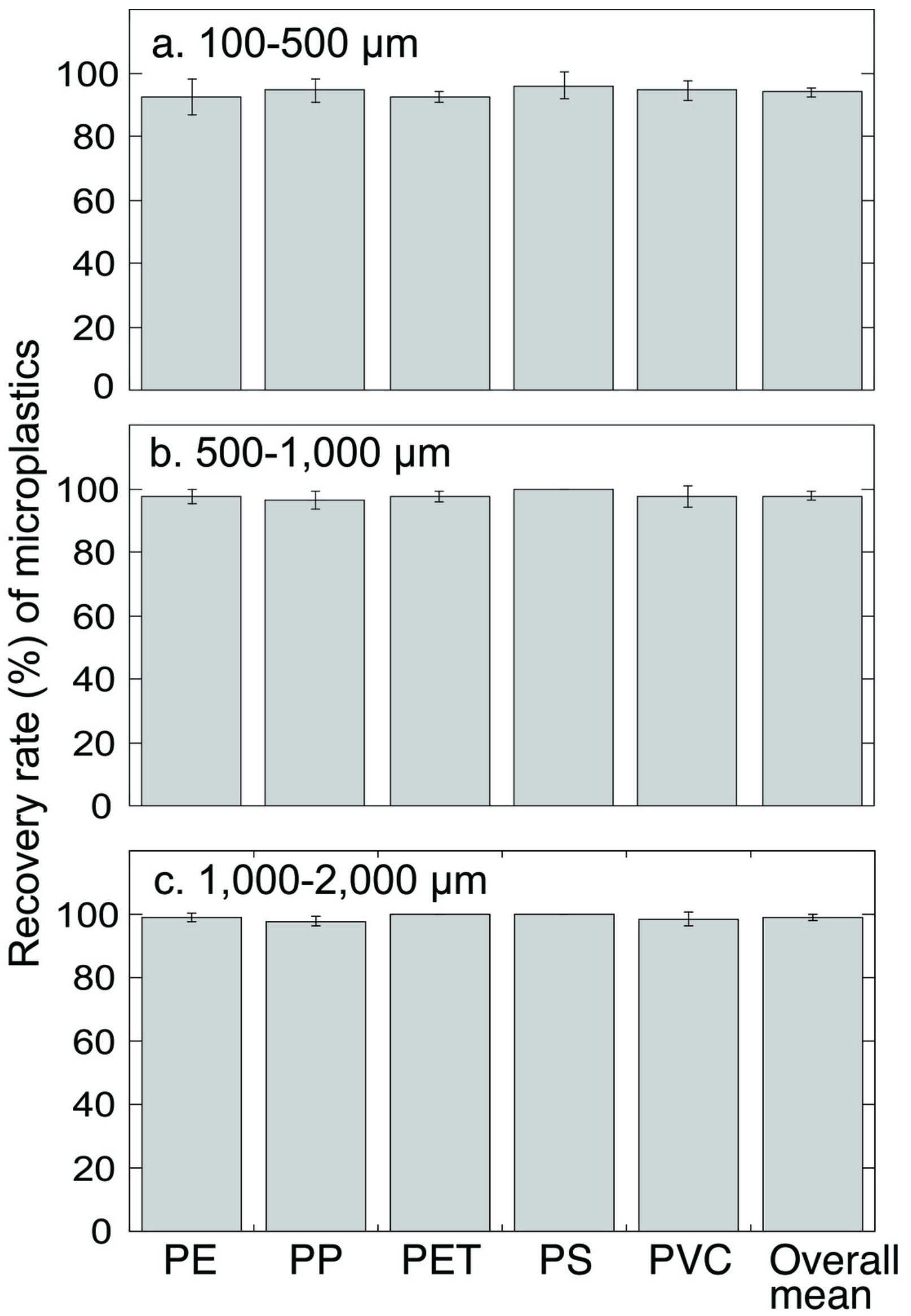


Figure 3

Recovery rate (\%) of microplastics by JAMSS unit using different sizes of sediment particles.

Each bar indicates percentage recovery rate of microplastics in different granularities of reef sediment (fine, medium, coarse). Error bars indicate standard deviation.

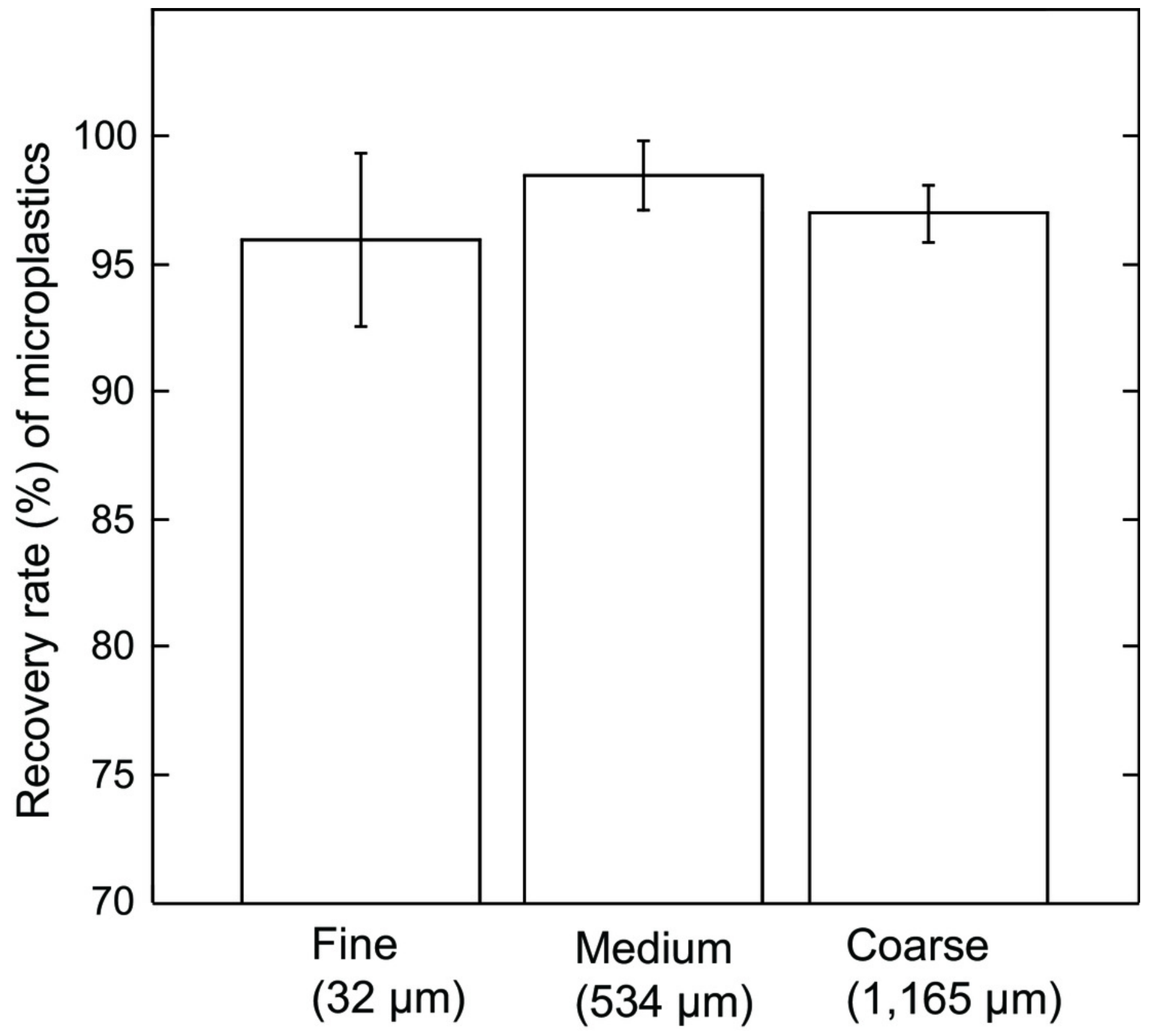

